\title{
Thymus: still an underexplored organ in medical practice
}

\author{
Magda M. S. Carneiro-Sampaio
}

\section{INTRODUCTION}

$\mathrm{T}$ he thymus remains one of the least explored organs in clinical practice, which can be explained, at least in part, because it is of difficult access for histopathological studies. On the other hand, there is growing evidence that the thymus may be affected both in congenital diseases and as a consequence of immunosuppressive medications, infections, nutritional disorders and several other clinical situations ${ }^{1,2}$. Until very recently, there were not many tests available for the functional assessment of the thymus. Its morphology, which was visualized through imaging techniques, and the study of subpopulations of peripheral T-cells were the only means to analyze indirectly the functional state of the organ. The advent of the technique for measuring the TRECs (T-cell receptor excision circles or thymic recent emigrant cells) in the peripheral blood has little more than a decade and is still restricted to research laboratories.

Much of what we know about the thymus comes from experimental studies, particularly those with murine and bird models. The increase in knowledge about the functions and disorders of the thymus in the last decades has been remarkable, and contrasts with the scarcity of data from human beings that allow practical progress in the identification and therapeutic approach of thymus diseases. HIV infection and the post-bone marrow transplant in neoplastic diseases have been the situations with more documented information on thymus function, in addition to the most recent interest in the aging process and means to promote its reversal or to delay it ${ }^{1,3}$. The identification of the Aire (autoimmune regulator) transcription factor, its role in the phenomenon of central tolerance and the discovery of a human disease caused by mutations in the AIRE gene also represent recent milestones in the understanding of the thymus ${ }^{4,5}$. The thymus was described about two thousand years ago by the renowned Roman physician Claudius Galen (130-200 AD), who was born in Pergamon, and is considered to be the father of Anatomy. The name "thymus" was given by Galen himself, because the organ (body) reminded him of thyme leaves (Thymus in Latin). It is worth clarifying that the plant was burned as incense. Throughout history and even until today, the organ is still wrapped in an aura of mystery, and there are indications that for the Greek the word thymus meant "active soul", the "perishable soul" and that it would be equivalent to reason and conscience. Only in 1961, with the experimental studies of the Australian physician Jacques Miller, consistent scientific data appeared, data that allowed for conclusions about its function, and that showed for the first time the thymus as a gland with a relevant role for the development of the immune system ${ }^{6}$.

Full Professor of the Department of Pediatrics of the University of São Paulo's Faculty of Medicine (FMUSP).

Mailing address: Instituto da Criança. Av. Dr. Enéas de Carvalho Aguiar, 647 - São Paulo, SP. ZIP CODE: 05403-900. Email: magdascs@usp.br 
In this paper, a brief review on the structure of the human thymus, its main functions, developmental aspects, means for its functional assessment and some thymic congenital diseases are presented.

\section{Functions of the Thymus}

The thymus is a primary lymphoid organ that plays a critical and unique role in the development of T-cells, providing the ideal microenvironment so that the precursors of lymphocytes derived from the bone marrow can proliferate, undergo the rearrangement of their receptor and so that their differentiation into mature T-cells can occur. When this differentiation occurs, these T-cells are able to establish an effective immune response, and then leave the thymus, go to the blood and to the peripheral or secondary lymphoid organs, that is, the spleen, lymph nodes and the mucosal associated lymphoid tissue ${ }^{8,9}$.

Another crucial (and also unique) function of the thymus is related to the induction of tolerance to the body's own antigens, thereby protecting it from autoimmune aggression. This function is represented by phenomena of: i) negative selection of potentially autoreactive T cells (central tolerance); and ii) generation of natural regulatory T cells (Treg), which will play their role especially outside the thymus ${ }^{7,8,9}$.

\section{Structure and development}

The thymus is essentially a lymphoepithelial organ and contains two anatomically and functionally distinct compartments, that is, the medulla, in the central part, surrounded by the cortex. Both compartments are formed by two major cellular components: i) the lymphoid component, rich in developing thymocytes and ii) the thymic stroma, consisting mainly of epithelial cells (Figure 1) ) $^{8,9,10}$. Other cells that are also present in the thymus are (the) dendritic cells, macrophages and B cells, and the role of the latter lineage is still not quite elucidated. The reciprocal interactions between epithelial cells and thymocytes are necessary for the development of both the lymphoid and the epithelial components of the thymus, resulting in the aforementioned functions. Thus, thymocytes release trophic factors essential to the development of the three-dimensional epithelial network, while the epithelial cells regulate the development of the T cells through the expression and presentation of the selfantigens by the major histocompatibility complex $(\mathrm{MHC})^{10}$. There are two examples of primary immunodeficiencies (PID) in which the thymus is not developed and that demonstrate very well the mutual interdependence between the lymphoid and epithelial components of the organ ${ }^{11}$. In severe combined immunodeficiencies (SCID), some mutations seriously harm the development of precursors of $\mathrm{T}$ cells, which fail to infiltrate the thymus during its development, while in DiGeorge syndrome (22q11.2 deletion syndrome), a defect in the development of the organ per se occurs ${ }^{12}$. In SCIDs, hematopoietic stem cell transplantation is the most effective therapy and induces the development of the epithelial component of the thymus after lymphoid reconstitution, while in the severe cases of DiGeorge syndrome, transplantation of the epithelial component of the thymus alone is enough to restore the immune system of the affected child ${ }^{13}$.

The development of the $\mathrm{T}$ cells is started by the migration of the so-called early thymic progenitors (ETP) from the bone marrow to the thymus ${ }^{8,14}$. The ETPs enter the thymus through the postcapillary venules of the corticomedullary junction, and begin to migrate towards the subcapsular region of the thymic cortex as double negative cells, that is CD4-CD8-. During the migration, the progenitor cells undergo differentiation resulting in the rearrangement and expression of its receptor ( $T$ cell receptor - TCR), and in the expression of the CD4 and CD8 components (double positive cells - DP, in the cortical region) of the receptor. After the expression of the TCR and of the co-receptors, the thymocytes are submitted to a positive selection process, during which the self-peptides presented by the MHC molecules on the cortical epithelial cells regulate the differentiation of only a small percentage of immature thymocytes so that they become mature T cells. The DP thymocytes whose TCR fail to interact or react with low affinity to the self-peptides-MHC complexes do not receive enough signals to survive and are eliminated through the process named death by neglect. "Positively" selected thymocytes, that is, those receiving the signal for survival in the cortex, migrate to the thymic medulla where they will undergo the so-called negative selection, whose 
main goal is to eliminate thymocytes which are potentially reactive against the body's own tissues, that is, autoreactive thymocytes. The thymocytes (here already as CD4 + or CD8 + single positives) displaying TCRs that have high or inappropriate affinity for the self-peptides (here tissuerestricted antigens - TRAs), whose expression is regulated by the previously mentioned Aire and which are presented by the epithelial cells are then eliminated within the thymic medulla. Thus, the consecutive positive selection and negative selection processes should originate an extensive repertoire of immunocompetent $T$ cells which is ideally free of autoreactive $\mathrm{T}$ cells and respond to non-self antigens. After a period of maturation subsequent to the selection, single positive $\mathrm{T}$ cells (CD4+CD8- and CD4-CD8+, whose TCRs will react with cells presenting Class I and Class II MHC, respectively) leave the thymic medulla and go straight to the periphery. In the thymic medulla there are structures that are quite typical of the organ, represented by the Hassall's corpuscles - CH (Figure 1), whose function remains to be fully clarified. The epithelial component of the thymus has its origin in the endoderm of the third pharyngeal pouch quite early in vertebrate ontogeny, which occurs around the sixth week of gestation in humans. The expression of the gene that codifies the Foxn1 (forkhead box protein N1) transcription factor in cells of the pharyngeal epithelium is considered to be the mark of the destination for the formation of the thymus ${ }^{9}$. The Foxn1 is essential for thymopoiesis and regulates the expression on the epithelial cells of key factors in the attraction of the hematopoietic precursors to the embryonic thymus, and infiltration by these precursors starts from the 60th day of gestation in humans on. The tissue differentiation of the thymus in cortex and medulla is completed between the 14th and 16th week of gestation, continuing its growth until it reaches the greater weight in relation to the body weight little before birth ${ }^{8}$. The organ continues to grow during the first months of extrauterine life and commonly reaches large volumes when compared to the rib cage in the first two to three years of life, representing the "big thymus" which is characteristic of infants and well-known by pediatricians (Figure 2) ${ }^{15}$. It continues to grow until adolescence, when it reaches its highest weight (about $40 \mathrm{~g}$ ), and from then on it begins to involute, with reduced size and weight of the cortical and medullary functional compartments (both the number of thymocytes and of epithelial cells), which are progressively replaced by adipose tissue. It is known that even during the process of involution, thymopoiesis continues to occur, originating new naïve $\mathrm{T}$ cells, even if in progressively smaller amounts, in particular after the 7 th and 8th decade of life ${ }^{3,16}$.

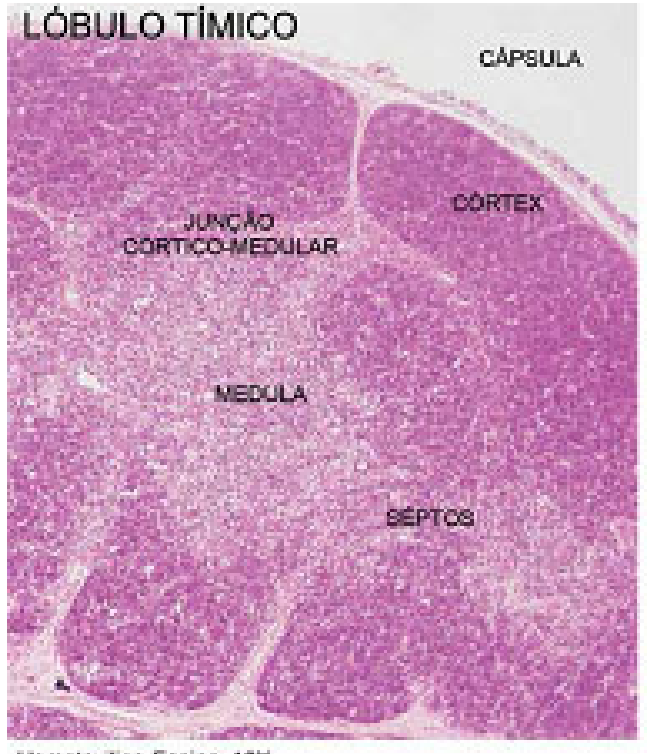

Hematocins-Eosins, $10 X$

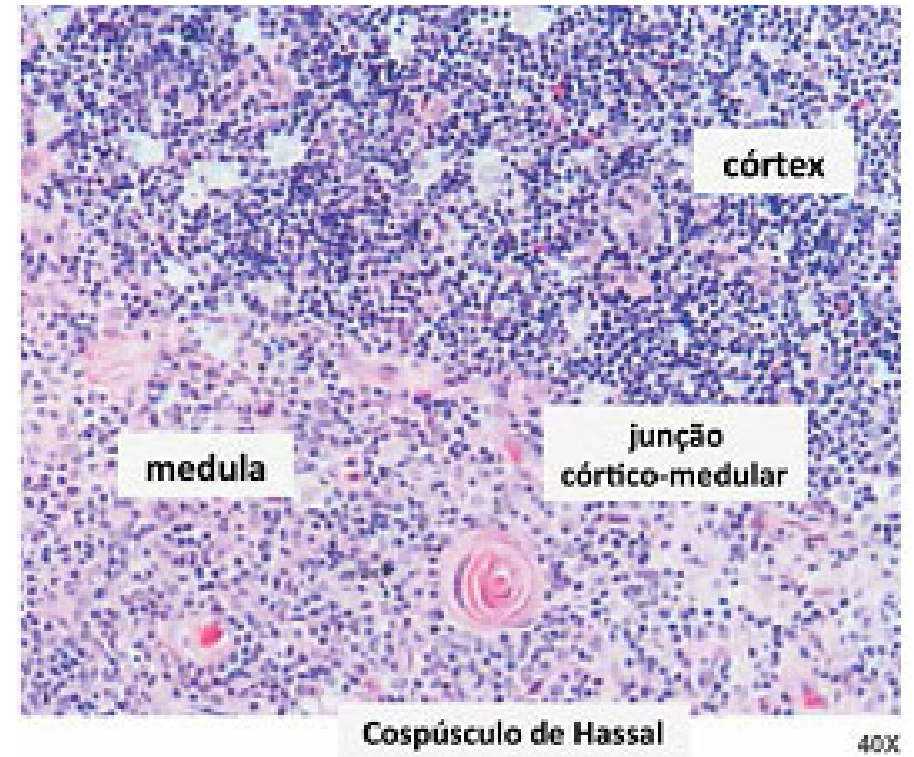

$40 \mathrm{X}$

Figure 1: Histological aspects of the thymus of a 10-month-old infant with surgical congenital heart defect (source: Lima ${ }^{30}$ ) 


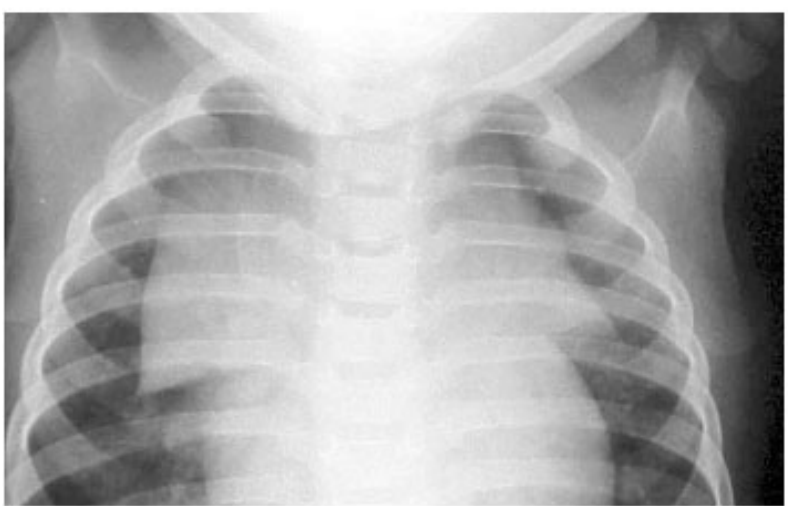

Figure 2: Image of a bilobed thymus seen on a chest radiograph of a 18-month-old eutrophic child, with acute viral respiratory infection (courtesy of the Children's Hospital Radiology Service, FMUSP Clinical Hospital)

\section{Assessment of thymic fuction}

In clinical practice, the methods for the assessment of thymic function are still incipient, restricted to research protocols, and has focused on: i) imaging methods that show the variations in the size of the organ. At the moment computed tomography is considered the best method, but its use in Pediatrics is quite limited because of the large amount of X-ray required to generate images Nowadays it would definitely not be acceptable from an ethical point of view to use computed tomography in protocols of research with children, and even in clinical practice, this technique should be restricted to situations where other potentially less damaging-to-the-tissues radiation (ultrasonography, conventional radiography and MRI) are not able to generate images with the same informative power ${ }^{17}$; ii) Assessing the absolute and percentage numbers of $\mathrm{T}$ cell populations in peripheral blood, particularly of the naïve cells (CD45RA+CD62L+CD27+), and since these cells can have a long life (thousand to 10 thousand days), its amount in peripheral blood does not reflect only the recent thymic output; iii) Measurement of the TRECs (T-cell receptor excision circles) in the peripheral blood, a technique developed in the late 1990s and proposed as a measure of thymic T cells output, is still far from being widely introduced into clinical practice ${ }^{16,18,19}$. The TRECs represent a product of the rearrangement of genes encoding the TCR of $\mathrm{T}$ lymphocytes during their intrathymic maturation process ${ }^{18}$. The TRECs are episomal circles which do not replicate during mitosis, and thus are diluted with each cell division. In this way, clonal expansion after the encounter with the antigen should reduce the content of TRECs + cells [of that(those) clone(s)] within the total pool of T cells in the peripheral blood. As the production of TRECs + naïve T cells is very low in other locations in the organism (intestines, for example), the number of TRECs+ naïve $\mathrm{T}$ cells has been considered as a reflex of the recent thymic production. However, in the interpretation of results, it is necessary to take into account, once more: i) the long lifespan of the naïve cells and ii) the speed of division of naïve cells in situations of infection, for example. The amount of TRECs + cells is very high in early life, especially in the first three years, it remains high until adolescence, when it starts to lower, coinciding with the process of thymic involution ${ }^{16,20}$. Thus, there is a temporal relationship between the great volumes of the thymus in the first two decades of life and the increased production of naïve T cells reflected the higher concentration TRECs in the peripheral blood $^{15}$. However, the doubt regarding the large thymuses seen in infants, whether they are functionally more active or not, remains. In other words, it is not known if the large thymuses release a greater number of TRECs + cells. They are always a reason to be concerned and require clinical and imaging monitoring. The parallel study of the TRECs could help in the clinical management of infants with "huge thymuses". On the other hand, the blood concentrations of TRECs have been demonstrated to be very low in the DiGeorge syndrome (Figure 3) and in severe combined immunodeficiencies (SCID), and even in Down syndrome, situations in which it is known that the thymus does not fully develop ${ }^{19}$.

\section{Some congenital diseases of the thymus}

Although in the daily clinical practice the hypothesis that the thymus may be affected is exceptionally rare, there is an increasing number of descriptions of primary diseases of the thymus, with some examples in the following paragraphs, but there are also numerous situations in which the thymus may be secondarily affected, such as: the use of immunosuppressive drugs (probably also corticosteroids), including here situations of organ and tissue transplants, several autoimmune diseases, severe infections, nutritional 
disorders, among other conditions which are common in clinical practice, especially among infants and children ${ }^{1,2,21}$.
The involution of the thymus during aging is also a process of great clinical interest $\mathrm{t}^{1,3}$.

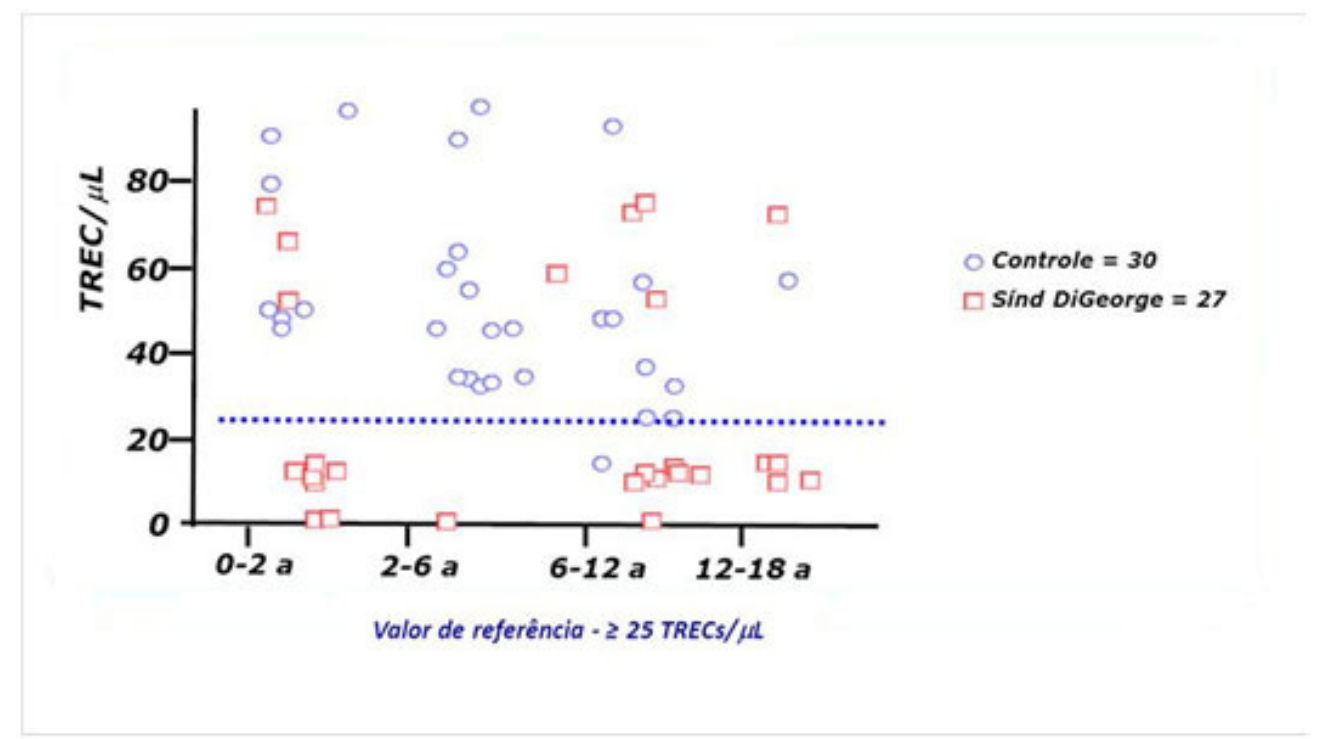

Figure 3. TRECs (T-cell receptor excision circles) blood levels from 27 patients with DiGeorge syndrome compared to age-matched healthy controls (data kindly provided by Dr. Leuridan Cavalcanti Torres)

\section{APECED (Autoimmune Polyendocrinopathy - Candidiasis - Ectodermal Dystrophy)}

The identification of this rare disease, whose acronym became its name, and which is also called autoimmune polyendocrine syndrome type 1 , stems from amorphic mutations in the AIRE gene, which encodes the autoimmune regulator transcription factor ${ }^{11}$. It is an autosomal recessive defect that has a higher frequency in the inhabitants of the island of Sardinia, Finland, and among Iranian Jews. The patients suffer from multiple autoimmune endocrinopathies, from which hypoparathyroidism (present in $88 \%$ of the cases in literature) and Addison's disease $(84 \%)$ are the most common, followed by hypogonadism (69\%), diabetes mellitus type I (33\%) and hypothyroidism $(31 \%)^{4}$. Chronic mucocutaneous candidiasis is present in all cases and is usually the first manifestation of the disease, which can already occur in the (very) first years of life. It is also currently considered as an autoimmune manifestation resulting from the action of anti-cytokine autoantibodies, in particular cytokine-neutralizing antibodies of the Th17 pathway (IL-17, IL-17A and IL-22) ${ }^{22}$. So far, the therapeutic approach to patients with APECED has focused on the treatment of candidiasis and of the autoimmune diseases, with the necessary hormone replacement ${ }^{4}$.

\section{Deficiency of the Foxn1}

The four patients described in literature with a mutation on the FOXN1 gene showed absence of thymus, undetectable or a very low number of $\mathrm{T}$ cells in the peripheral blood (equivalent to those of SCID), the absence of hair and eyebrows, nail dysplasia, in addition to an increased susceptibility to infections ${ }^{23}$. One of the most recently reported cases is that of a Portuguese child who received thymic epithelium transplant with a quite satisfactory immunological reconstitution, however the description is that the child remains in complete alopecia. It is the human equivalent to the nude mouse, and this rare "experiment of nature" brings unique information on the key role of the Foxn1 transcription factor also for the development of the human thymus.

\section{2q11.2 Deletion Syndrome (SD22q11.2)}

SD22q11.2 [also called DiGeorge Syndrome (DGS) when associated to thymic alterations] is considered as the most common chromosomal microdeletion in humans. It is estimated that this syndrome happens at a frequency of 1 : 4,000 live births, which means that about 150 children with this condition are born each year in the State of São Paulo 
(about 600,000 births/year) ${ }^{19,24}$. The most common clinical manifestations in SD22q11.2 are: congenital heart defects, particularly defects of the great vessels (75-80\% of cases), hypocalcemia due to agenesia of the parathyroid glands (60\%), facial dysmorphisms (Figure 4) and velopharyngeal insufficiency (46\%) and immunological alterations, which vary greatly among those affected, resulting from thymus development disorders. It is estimated that in 1 to $2 \%$

A

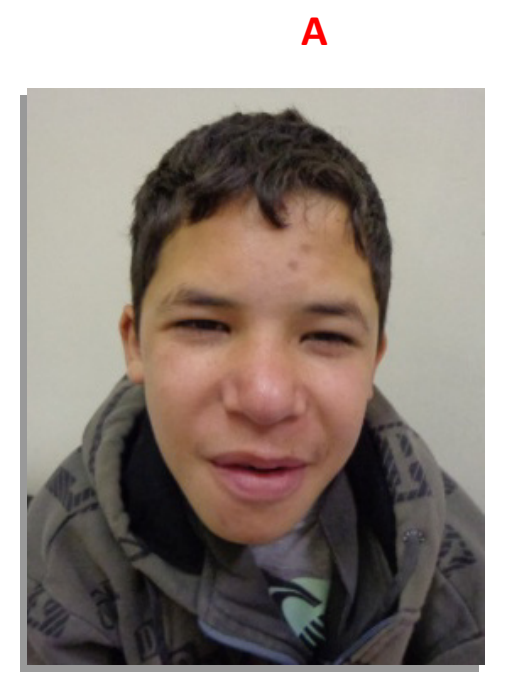

of cases thymic agenesia occurs, and in the remaining cases there is the occurrence of hypoplasia of varying degrees ${ }^{12,19,24}$. Figure $4 \mathrm{C}$ shows a chest radiograph of an infant with DGS, where a thymic image cannot be observed, while Figure 3 presents the TRECs levels in some of the patients of the Children's Institute, compared with controls of the same age, represented by patients who underwent small elective surgeries.
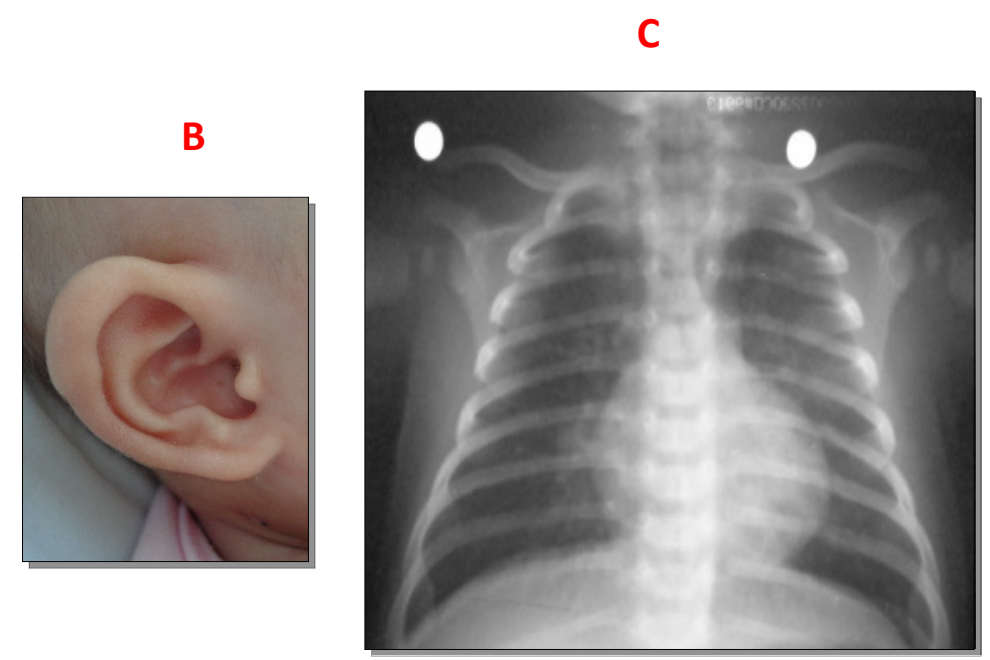

Figura 4. Main phenotypic characteristics of patients with the DiGeorge syndrome: A) typical facies with narrow palpebral fissure, eyelid hooding, hypertelorism, square nose, elongated nose and face; B) infant with low implantation and dysplasic overfolded ears helix; C) the chest X-ray shows absence of thymic image (pictures kindly provided by Dr. Marcilia Sierro Grassi)

Despite the large number of cases already studied with DGS, it has not been possible to establish clear relationships between the clinical phenotype and the size of the chromosomal deletion ${ }^{24}$. Neither has it been possible to establish relationships between the size of the deletion and the degree of impairment of the thymic development and, therefore, with the severity of the immunodeficiency in the affected patients. Classic findings of the DGS have been described in patients with mutations in the TBX1 gene (encoded at the site of deletion), as well as malformations equivalent to that of this disease have been observed in knock-out mice for this gene. The immune deficiency observed in these patients is not only due to the quantitative deficiency of $\mathrm{T}$ cells as well as to abnormalities in the repertoire of $\mathrm{T}$ cells and reduction in their proliferation activity. Moreover, some patients present impairment of humoral immunity, with significant antibody disfunction. Autoimmune diseases, especially cytopenias, are not uncommon in DGS patients, and are more frequent than in the overall population ${ }^{11}$. The evaluation of humoral immunity in DGS has revealed that about $6 \%$ of those affected have significant hypogammaglobulinemia, requiring gamma-globulin replacement therapy ${ }^{25}$.

\section{Down Syndrome (DS)}

The thymus of those affected by DS has been drawing attention since the 1970s, and has been described as having small dimensions, even in newborns and infants. The thymus presents an abnormal structure with some frequently observed histological changes, such as general hypocellularity, lack of clear demarcation between the cortical and medullary regions, narrowing of the cortex, huge Hassall corpuscles for one's age), among other changes ${ }^{26,27,28,29}$. The TRECs concentrations have been presented as lower in peripheral blood when compared with age-matched controls and demonstrate the hypofunction of the thymus in patients with trisomy 21 . Our research 
group at Instituto da Criança (HC-FMUSP) observed a low expression of the AIRE protein and gene (encoded on 21q22.3) in thymuses of children with $\mathrm{DS}^{28}$. These patients have, in fact, some phenotypic characteristics in common with APECED: i) high frequency of organ-specific autoimmune diseases, especially endocrinopathies (in DS, especially hypothyroidism and diabetes mellitus type 1); ii) increased susceptibility to mucocutaneous candidiasis; and iii) the presence of autoantibodies which are unique to APECED. In this study, the reduced expression of Aire was observed both in infants and in older children, and the differences between patients and controls (patients with congenital heart disease (such) as an isolated malformation) were significantly higher (bigger) in patients (children) younger than one year of age. These results strongly suggest that the thymic alterations in DS are not caused by senescence of the immune system, but rather are present from the very beginning of development, and therefore must be caused by an intrinsic defect ${ }^{28}$.

More recently, our group was able to characterize, in the human thymus, the transcriptional changes induced by trisomy 21 through the analysis of gene coexpression networks (GCNs) and microRNA-target interactions ${ }^{29}$. In this study we used cortical and medullary explants from thymic tissue - obtained in cardiac surgery in patients with DS and karyotipically normal controls (KNC) - and an approach to GCNs analysis that allows the identification of the repertoire of transcriptional modules (communities) and interactions between all the components of the system by detecting communities. Alterations in the degree observed connections for hubs/hierarchically important genes in $\mathrm{KNC}$ and DS networks corresponded to alterations in communities. The role of the miRNAS in modulationg the expression of highly connected genes in KNC and DS was revealed through the analysis of the miRNA-target interactions. The gene disruption caused by trisomy 21 in the human thymus can be described as the disruption and altered reorganization of transcriptional modules. The leading GCNs acting in the normal state (KNC) and altered state (DS) of the thymus were identified. In summary, the KNC networks represent the thymus' canonical way of functioning. On the other hand, the DS networks represent a non-canonical way, that is, adaptation of the thymic tissue under the gene disruption caused by trisomy 21 . This adaptation is probably driven by epigenetic mechanisms occurring at the chromatin level and through miRNAs that control transcriptional programs involving high-hierarchy genes of the GCNs.

ACKNOWLEDGMENTS: The functional study of the thymus at the Children's Hospital of the FMUSP Academic Medical Center has received financial support from FAPESP (thematic project 2014/50489-9).

\section{REFERENCES}

1. Holländer GA, Krenger W, Blazar BR. Emerging strategies to boost thymic function. Curr Opin Pharmacol. 2010;10:443-53. doi: 10.1016/j.coph.2010.04.008.

2. Nunes-Alves C, Nobrega C, Behar SM, Correia-Neves M. Tolerance has its limits: how the thymus copes with infection. Trends Immunol. 2013;34:502-10. doi: 10.1016/j. it.2013.06.004.

3. Boehm T, Swann JB. Thymus involution and regeneration: two sides of the same coin? Nat Rev Immunol. 2013;13:8318. doi: $10.1038 /$ nri3534

4. Arstila TP, Jarva H. Human APECED; a Sick Thymus Syndrome? Front Immunol. 2013;7;4:313. doi: 10.3389/ fimmu.2013.00313.

5. Mathis D, Benoist C. Aire. Annu Rev Immunol. 2009;27:287312. doi: 10.1146/annurev.immunol.25.022106.141532.

6. Miller JF. Immunological function of the thymus. Lancet. 1961;2(7205):748-9. doi:10.1016/S0140-6736(61)90693-6.
7. Coutinho A, Caramalho I, Seixas E, Demengeot J. Thymic commitment of regulatory $\mathrm{T}$ cells is a pathway of TCRdependent selection that isolates repertoires undergoing positive or negative selection. Curr Top Microbiol Immunol. 2005;293:43-71

8. Lima, FA, Carneiro-Sampaio M. O papel do timo no desenvolvimento do sistema imune. Pediatria (São Paulo). 2007;29:33-42.

9. Miller JF. The golden anniversary of the thymus. Nat Rev Immunol. 2011;11(7):489-95. doi: 10.1038/nri2993.

10. Alexandropoulos K, Danzl NM. Thymic epithelial cells: antigen presenting cells that regulate $\mathrm{T}$ cell repertoire and tolerance development. Immunol Res. 2012;54:177-90. doi: 10.1007/s12026-012-8301-y.

11. Carneiro-Sampaio M, Coutinho A. Tolerance and autoimmunity: lessons at the bedside of primary immunodeficiencies. Adv Immunol. 2007;95:51-82. doi: 10.1016/S0065-2776(07)95002-6. 
12. Gennery AR. Immunological features of 22q11 deletion syndrome. Curr Opin Pediatr. 2013;25:730-5. doi: 10.1097/ MOP.0000000000000027

13. Markert ML, Devlin BH, McCarthy EA. Thymus transplantation. Clin Immunol. 2010;135:236-46. doi: 10.1016/j.clim.2010.02.007.

14. Sauce D, Appay V. Altered thymic activity in early life: how does it affect the immune system in young adults? Curr Opin Immunol. 2011;23:543-8. doi: 10.1016/j.coi.2011.05.001.

15. Carneiro-Sampaio MMS, Zago C, Palmeira P. Desarrollo de la respuesta inmune. In: Quezada Lagos A, Araos DZ. Alergia e inmunologia respiratoria. Santiago, Chile: Ed Mediterraneo; 2013. p.32-42.

16. Zhang L, Lewin SR, Markowitz M, Lin HH, Skulsky E, Karanicolas R, He Y, Jin X, Tuttleton S, Vesanen M, Spiegel H, Kost R, van Lunzen J, Stellbrink HJ, Wolinsky S, Borkowsky W, Palumbo P, Kostrikis LG, Ho DD. Measuring recent thymic emigrants in blood of normal and HIV-1-infected individuals before and after effective therapy. J Exp Med. 1999;190:725-32. doi: 10.1084/ jem.190.5.725.

17. Valente M, Oliveira LAN, Carneiro-Sampaio, M. Radiologia pediátrica: quando o diagnóstico deve ser "amigo" da criança [editorial]. Radiol Bras. 2012;45(5):v. http://dx.doi.org/10.1590/S0100-39842012000500001.

18. Hazenberg MD, Borghans J, de Boer RJ, Miedema F. Thymic output: a bad TREC record. Nat Immunol. 2003;4:97-9. doi: 10.1038/ni0203-97.

19. Grassi MS, Jacob CMA, Kullikovski LD, Pastorino AC, Dutra RL, Miura N, Jatene MB,Pegler SP, Kim AE, , Carneiro-Sampaio M. Congenital heart disease as a warming sign for the diagnosis of the 22q11.2 deletion. Arq Bras Cardiol. 2014;103(5):382-390. doi: 10.5935/ abc. 20140145 .

20. Arismendi MI, Kallás EG, Santos BA, Carneiro-Sampaio MM, Kayser C. Thymopoiesis and regulatory $\mathrm{T}$ cells in healthy children and adolescents. Clinics (Sao Paulo). 2012;67(5):425-9. doi: 10.6061/clinics/2012(05)04.

21. Horvath D, Kayser C, Silva CA, Terreri MT, Hilário MO, Andrade LE. Decreased recent thymus emigrant number in rheumatoid factor-negative polyarticular juvenile idiopathic arthritis. Clin Exp Rheumatol. 2010;28(3):348-53. Available from: http://www.clinexprheumatol.org/article.asp?a=295.

22. Kisand K, Boe Wolff AS, Podkrajsek KT, Tserel L, Link M, Kisand KV, et al. Chronic mucocutaneous candidiasis in APECED or thymoma patients correlates with autoimmunity to Th17-associated cytokines. J Exp Med. 2010;207:299-308. doi: 10.1084/jem.20091669.

23. Markert ML, Marques JG, Neven B, Devlin BH, McCarthy EA, Chinn IK, Albuquerque AS, Silva SL, Pignata C, de Saint Basile G, Victorino RM, Picard C, Debre M, Mahlaoui N, Fischer A, Sousa AE. First use of thymus transplantation therapy for FOXN1 deficiency (nude/SCID): a report of 2 cases. Blood. 2011;117:688-96. doi: 10.1182/ blood-2010-06-292490.

24. Kobrynski LJ, Sullivan KE. Velocardiofacial syndrome, DiGeorge syndrome: the chromosome 22q11.2 deletion syndromes. Lancet. 2007;370(9596):1443-52. doi: 10.1016/ S0140-6736(07)61601-8.

25. Patel K, Akhter J, Kobrynski L, Benjamin Gathmann MA, Davis O, Sullivan KE; International DiGeorge Syndrome Immunodeficiency Consortium. Immunoglobulin deficiencies: the B-lymphocyte side of DiGeorge Syndrome. J Pediatr. 2012;161(5):950-3. doi: 10.1016/j. jpeds.2012.06.018.

26. Larocca LM, Lauriola L, Ranelletti FO, Piantelli M, Maggiano N, Ricci R, Capelli A. Morphological and immunohistochemical study of Down syndrome thymus. Am J Med Genet Suppl. 1990;7:225-30.

27. Kusters MA, Verstegen RH, Gemen EF, de Vries E. Intrinsic defect of the immune system in children with Down syndrome: a review. Clin Exp Immunol. 2009;156(2):18993. doi: $10.1111 / \mathrm{j} .1365-2249.2009 .03890$.x.

28. Lima FA, Moreira-Filho CA, Ramos PL, Brentani H, Lima LA, Arrais M, Bento de Souza LC, Bento de Souza L, Duarte MIS, Coutinho A, Carneiro-Sampaio M. Decreased AIRE expression and thymic hypofunction in Down syndrome. J Immunol. 2011;187:3422-30. doi: 10.4049/ jimmunol.1003053.

29. Moreira-Filho CA, Bando SY, Bertonha FB, Silva FN, Costa LD, Ferreira LR, Furlanetto G, Chacur P, Zerbini MC, Carneiro-Sampaio M. Modular transcriptional repertoire and MicroRNA target analyses characterize genomic dysregulation in the thymus of Down syndrome infants. Oncotarget. 2016;7(7):7497-533 doi: 10.18632/ oncotarget. 7120

30. Lima FA. Estudo da expressão da proteína Aire (autoimune regulator) e dos componentes da via de sinalização Notch em timos humanos [tese]. São Paulo: Instituto de Ciências Biomédicas, Universidade de São Paulo; 2011. Available from: http://www.teses.usp.br/teses/disponiveis/42/42135/ tde-04082011-150437/pt-br.php. 